\title{
KN13 | Mesophase Mirabilis. The Lipid Cubic Phase As A System For Investigating
}

\section{Membrane Proteins}

Caffrey, Martin (Trinity College Dublin, Dublin 4)

Membrane Structural and Functional Biology Group, School of Medicine and School of Biochemistry and Immunology, Trinity Biomedical Sciences Institute, Trinity College Dublin, Dublin 2, Ireland.

The lipid cubic phase (in meso) method for crystallizing membrane proteins has to its credit close to 700 structure records in the Protein Data Bank. Progress in applying the method reflects innovations that relate to protein enrichment, direct biophysical and biochemical characterization and refolding in the mesophase and in situ data collection at X-ray synchrotrons and free electron lasers using microcrystals. These varied methods will be reviewed and examples will be provided where they have contributed to the determination of high-resolution crystal structures of membrane integral enzymes, receptors, transporters and carriers with insights into mechanism of action and the prospect of drug discovery.

Funded in part by Science Foundation Ireland. 